\title{
Developing novel methods for protein, peptide and metabolite analysis
}

\author{
Rainer Bischoff*,1 \\ ${ }^{1}$ Analytical Biochemistry, Department of Pharmacy, University of Groningen, Antonius Deusinglaan 1, 9713 AV Groningen, The \\ Netherlands \\ * Author for correspondence: r.p.h.bischoff@rug.nl
}

Rainer Bischoff, Professor of Analytical Biochemistry at the University of Groningen (Groningen, The Netherlands), talks to Sankeetha Nadarajah, Managing Commissioning Editor (Bioanalysis), regarding the development of novel methods for protein, peptide and metabolite analysis.

Accepted for publication: 15 November 2017; Published online: 10 April 2018

\section{Can you describe the major approaches that you use? What are their possibilities \& the current challenges?}

We use LC-MS at all levels, from normal flow $(0.2 \mathrm{ml} / \mathrm{min})$ all the way to chip-based systems and nanoflow. The challenges, I think, are very much related to sample preparation because we work with complex biological samples such as blood-based materials or tissue biopsies, so you have a large amount of protein. Within this myriad of proteins, it is quite difficult to detect a specific protein, especially if the protein is of low abundance. For blood plasma or serum, you need significant sample preparation, to reach the nanogram per milliliter level. That means that we focus a lot on sample preparation in combination with validation of the method.

There are a number of challenges, especially for the biomarker world, related to the internal standard or reference materials. For many endogenous proteins, it is not possible to get good protein standards. Recombinant proteins, from our experience, often do not match endogenous proteins exactly, leading to different recoveries and so on, which cannot be easily corrected. The main challenge for LC-MS compared with Ligand Binding Assays (LBA) is sensitivity but the advantage is the chemical information that you get.

Other methodologies that we use are gel electrophoresis, Western blots and all kinds of biochemical methods.

\section{Considering the challenges in the development of specific antibodies, can you give us your thoughts on antibody-free LC-MS/MS \& what role it has in protein bioanalysis?}

For the time being, I do not think they play a major role, because antibody-based assays are immunocapture assays, which are presumably easier to develop. However, you always depend on the specificity of the antibody. For many antibodies, specificity is not disclosed, so you are almost working in a 'black box' type of a system. The research community has realized this and there are strong efforts in academic as well as in industrial research to only work with affinity reagents where the binding site (epitope) and even the whole sequence are disclosed.

The challenge for antibody-free assays is that you need to reach the same sensitivity as for antibody-based assays. Antibody-free assays are very good to complement antibody-based assays, notably to check what you are actually measuring. If you are working with plasma or serum, you need to enrich proteins across six to nine orders of magnitude without antibodies in a way that is specific enough to reach (sub) nanogram per milliliter concentrations. We have done this in a couple of cases, where we have, for example, used isoelectric points, hydrophobicity and/or metal-binding capabilities to reach sub-nanogram per milliliter concentration levels. In the literature, you can also find nice examples where people have used hydrophobicity and precipitation for protein enrichment.

The development of antibody-free protein enrichment methods depends on an excellent understanding of the physical-chemical properties of the target protein(s) and the protein-rich matrix. There is no 'one-size fits all method', so you have to look at the physical-chemical properties of your protein, compare it with the matrix, and then try to find differences and from there on develop the enrichment method. I would not say it works in every case, but that is also true of antibody-based assays where you may have cross-reactivity and other difficulties. 
I think it is good to have different kinds of assays, including those that do not rely on antibodies to 'cross-validate' the results. Unfortunately, in the biomarker field, there is only a single, commercially available ELISA with dozens of clinical studies that all use this one ELISA. This may lead to problems, such as a systematic bias and the risk that, should the assay disappear due to quality or production issues, there is no possibility for an entire research area to continue. So we have often been asked by our clinical chemistry colleagues to develop, for example, an MS-based assay as a reference method for an LBA.

\section{What further advances do you envisage in this field over the coming years?}

The development of affinity binders, not only antibodies but also DARPINs, AFFIMERs and other smaller proteins like nanobodies or nucleic acid-based binders, where the chemical entity is defined, will enter the field of LBAs and affinity-MS assays. I think we will move beyond the time where manufacturers do not disclose what the epitope of an antibody is and professionals have to work in the dark. I do hope this will change soon.

I think we will also see more multiplex assays of panels of proteins, especially in the biomarker field. I think we all agree that multifactorial common diseases cannot be diagnosed or prognosticated with just one protein or just one metabolite, so we will also see proteins and metabolites measured together with other compounds such as microRNAs. As the field is expanding, it will require not only new methods but also new data analysis approaches because the classical univariate statistical analytical approaches will be replaced by multivariate statistical tools.

High-resolution mass spectrometry (HRMS) will be more commonly used in regulated bioanalysis, a development that has already started. Although HRMS has advantages, it is still a new technique in this field, so robustness and so on must still be checked. But instrument development is going into that direction, so it is a matter of time when we will see more HRMS instruments in routine laboratories.

Disclaimer

The opinions expressed in this interview are those of the interviewee and do not necessarily reflect the views of Future Science Ltd.

Financial \& competing interests disclosure

The author has no relevant affiliations or financial involvement with any organization or entity with a financial interest in or financial conflict with the subject matter or materials discussed in the manuscript. This includes employment, consultancies, honoraria, stock ownership or options, expert testimony, grants or patents received or pending, or royalties.

No writing assistance was utilized in the production of this manuscript. 